\title{
Qubit as the natural unit measure of random choice
}

\author{
Vasil Penchev, vasildinev@gmail.com \\ Bulgarian Academy of Sciences: Institute of Philosophy and Sociology: \\ Dept. of Logical Systems and Models
}

\begin{abstract}
Einstein wrote his famous sentence "God does not play dice with the universe" in a letter to Max Born in 1920. All experiments have confirmed that quantum mechanics is neither wrong nor "incomplete". One can says that God does play dice with the universe. Let quantum mechanics be granted as the rules generalizing all results of playing some imaginary God's dice. If that is the case, one can ask how God's dice should look like. God's dice turns out to be a qubit and thus having the shape of a unit ball. Any item in the universe as well the universe itself is both infinitely many rolls and a single roll of that dice for it has infinitely many "sides". Thus both the smooth motion of classical physics and the discrete motion introduced in addition by quantum mechanics can be described uniformly correspondingly as an infinite series converges to some limit and as a quantum jump directly into that limit. The second, imaginary dimension of God's dice corresponds to energy, i.e. to the velocity of information change between two probabilities in both series and jump.
\end{abstract}

Key words: Einstein vs quantum mechanics, Gleason's theorem, Fermat's last theorem, Kolmogorov's axiom of probability, qubit

\section{INTRODUCTION}

Prehistory: Einstein wrote his famous sentences in a letter to Max Born dated "16 December 1926":

Die Quantenmechanik ist sehr achtunggebietend. Aber eine innere Stimme sagt mir, daß das noch nicht der wahre Jakob ist. Die Theorie liefert viel, aber dem Geheimnis des Alten bringt sie uns kaum näher. Jedenfalls bin ich überzeugt, daß der nicht würfelt ${ }^{1}$ (Einstein 1926).

They synthesize Einstein's resistance to quantum mechanics in a few phrases. Nevertheless, all experiments have confirmed that quantum mechanics is neither wrong nor "incomplete" ${ }^{2}$ (Bell 1964;

1 "Quantum mechanics is very worthy of respect. But an inner voice tells me that that's not the real McCoy. The theory says a lot, but brings us hardly closer to the secret of the Old. Anyway, I am convinced that he does not play dice."

${ }^{2}$ The alleged "incompleteness" of quantum mechanics is the subject and title of the so-called EPR article (Einstein, Podolsky, and Rosen 1935). In fact, the three authors forecast theoretically the phenomena of entanglement, however, in a way of "reductio ad absurdum": The suggested completeness of quantum mechanics implies those phenomena, and as ostensibly they were nonsense, 
Clauser and Horne 1974; Aspect, Grangier, and Roger 1981; 1982). Quantum mechanics is true: "the truth and the whole truth". One can says that God does play dice with the universe.

However, these phrases of Einstein have generated nowadays a new scientific area ${ }^{3}$, "quantum dicelogy", said not too seriously. It might feature as an interdisciplinary field between probability theory, quantum mechanics, information theory, ontology, epistemology, and even theology. It would include history and philosophy of science as well as popular representations of quantum mechanics and Einstein's polemic with it.

However, almost all publications consider the image of those "dice" as a metaphor for quantum indeterminism and related topics. On the contrary, the present discussion considers literally like what they should look from a properly scientific viewpoint.

The origin of that idea can be traced even still to the Sixth Problem of Hilbert (1900):

Durch die Untersuchungen über die Grundlagen der Geometrie wird uns die Aufgabe nahegelegt, nach diesem Vorbilde diejenigen physikalischen Disziplinen axiomatisch zu behandeln, in denen schon heute die Mathematik eine hervorragende Rolle spielt; dies sind in erster Linie die Wahrscheinlichkeitsrechnung und die Mechanik ${ }^{4}$.

It has been very often considered as a problem, which is not properly mathematical unlike the rest 22 ones. One can stress the prophetic gift of Hilbert as he unifies probability theory, mechanics ${ }^{5}$, on the one hand, and on the other hand, geometry and mathematics in an axiomatic and deductive way in the entire context of his Sixth Problem. Indeed their unification would show that mechanics (and by its meditation both physics and natural science) and mathematics would link to each other very closely and thus passing into each other gradually and continuously without any gap of dualism:

Still since the age of Galileo and Newton, physics has been described by mathematically formulated laws. However the cause for this was possible remained mysterious and metaphorically explained as the sentence: "Mathematics is the language of nature".

The problem:

Let quantum mechanics be granted as the rules generalizing all results of playing some imaginary God's dice. If that is the case, one can ask how God's dice should look like. This means the following in mathematical and physical terms:

If all possible experimental results on a single quantum system can be represented by its wave function, which in turn is some point in Hilbert space, what is the elementary choice determining any single result unambiguously? In other words, what is the elementary event if the space of all events

it should be "incomplete". Schrödinger (1935) forecast independently them under the name of "verschränkte Zustände" without the label of "absurd".

${ }^{3}$ Chamberlain 1987; Ponomarev 1993; de la Peña and Cetto 1996; Koperski 2000; Saunders 2000; Stewart 2002; Floit 2007; Breshears 2008; Shiang 2008; Montwill and Breslin 2012; Bianchi 2013; Clegg 2014; Halpern 2015; and many, many others. There are conferences and $\mathrm{PhD}$ theses about those "dice" as well.

4 "The investigations on the foundations of geometry suggest the problem: To treat in the same manner, by means of axioms, those physical sciences in which mathematics plays an important part; in the first rank are the theory of probabilities and mechanics" (Hilbert 1902: 454).

${ }^{5}$ Quantum mechanics was to be outlined in decades later than 1900 when Hilbert declared his 23 Problems. 
consists of all possible experimental results, which can be obtained for that system, in the sense of probability theory and Kolmogorov's axioms (1933)?

One can visualize the problem by usual dice: any roll of the dice is both an elementary event and choice between six equally probable alternatives usually designated by $1,2,3,4,5$, and 6 . How many of different symbols would necessary be for "each side" of God's dice?

The thesis: God's dice is a qubit ${ }^{6}$. This means that the solving of the problem is the normed superposition of two subspaces of the complex Hilbert space called a quantum bit (or qubit).

A qubit $=\alpha|0\rangle+\beta|1\rangle$, where $\alpha, \beta$ are two complex numbers so that $|\alpha|^{2}+|\beta|^{2}=1$, and $|0\rangle,|1\rangle$ are two different subspaces of the complex Hilbert space.

If one utilizes the above analogy, God's dice need an infinite set of different symbols for all sides of them.

\section{A SKETCH OF THE PROOF OF THE THESIS:}

Any point of Hilbert space (i.e. any wave function) can be represented an infinite series of qubit: that is an infinite series of "God's rolls" of those dice having an infinite number of sides. Indeed any point in the complex Hilbert space is a vector:

$C_{1}, C_{2}, C_{3}, \ldots, C_{n}, C_{n+1}, \ldots$, where " $n$ " is a positive integer; and $C_{n}$ is a complex number.

One can construct an equivalent series of qubits:

$Q_{1}, Q_{2}, C_{3}, \ldots, Q_{n}, Q_{n+1}, \ldots$, where " $n$ " is a positive integer, and $Q_{n}$ is a qubit, if $\alpha_{n}, \beta_{n}$ for $Q_{n}$ are determined as follows:

$$
\begin{gathered}
\alpha_{n}=\frac{C_{n}}{+\sqrt{\left|C_{n}\right|^{2}+\left|C_{n+1}\right|^{2}}} ; \beta_{i}=\frac{C_{n+1}}{+\sqrt{\left|C_{n}\right|^{2}+\left|C_{n+1}\right|^{2}}} \text { if } C_{n}, C_{n+1} \text { are not both } 0 ; \\
\alpha_{n}=0 ; \beta_{n}=0 \text { if } C_{n}, C_{n+1} \text { are both } 0, \text { as a convention. }
\end{gathered}
$$

The convention is chosen meaning the consideration in Section V. Furthermore, $|0\rangle_{n}=e^{i n \omega}$, and $|1\rangle_{n}=e^{i(n+1) \omega}$, where $i$ is the imaginary unit, and $\omega$ is angular frequency corresponding to the unit distance between two "axes" in the complex Hilbert space.

Consequently any vector of $n+1$ complex numbers is equivalent to a series of $n$ qubits: that is a series of $n$ "rolls" of God's dice. If $n+1=\infty, n=\infty$, too.

The "rolls" are independent of each other for any two qubits constructed as above are orthogonal to each other.

One can easily check out that Kolmogorov's axioms $(1933: 2,13)$ for probability are satisfied:

1. All sets of rolls constitute algebra of sets $\mathfrak{I}$.

2. That algebra of sets $\mathfrak{I}$ includes the set $E$ of all rolls.

\footnotetext{
${ }^{6}$ Nonetheless that qubit is a single one, one should use "dice" (plural) rather than a "die" (singular) for any qubit means two independent choices and thus a pair of dice. Each of the orthogonal subspaces, $|0\rangle$ and $|1\rangle$, can be interpreted as a single die of that pair of dice, and the complex numbers $\alpha$ and $\beta$ as the symbols (numerals), which are "fallen" in a given roll of God's dice. This more accurate definition is fundamentally important as Section V will elucidate.
} 
3. and 4. $\left|\alpha_{n}\right|^{2}$ is a real number, which is less or equal to 1 , and thus it can be interpreted as the probability associable with the event for the $n$ roll to be $Q_{n}$. Then, $\left|\beta_{n}\right|^{2}$ would be interpretable as the probability that $Q_{n}$ does not occur, and $\left|\alpha_{n}\right|^{2}+\left|\beta_{n}\right|^{2}=1$. This is a trivial corollary from the so-called Born (1926) rule for the squire of the module of wave function to be interpreted as the probability of measuring or observing the events, to which this wave function refers.

4. The probability of all rolls $P(E)$ is 1 for the wave function is normed: $\Psi . \Psi^{*}=1$.

5. Indeed $P(A+B)=P(A)+P(B)$ as all rolls are independent of each other because any two qibits in the above representation of wave function are orthogonal to each other.

6. The axiom of continuity is implied by the continuity of wave function. The sixth axiom of Kolmogorov is necessary since the space of events (rolls) as well as the set $E$ and algebra $\mathfrak{I}$ are infinite. Not being infinite, Kolmogorov demonstrated that Axiom 6 is a conclusion from the axioms 1-5 (1933: 13-14).

The axiom 5 and 6 as to any infinite space of events can be unified and represented as an interpretation of Kolmogorov's theorem (1933: 14): The probability of any countable union of rolls is the sum of the probabilities corresponding to each roll for the rolls are always independent of each other.

\section{GLEASON'S THEOREM (1957) AND THE THESIS:}

However, a qubit falls just into the exception of two dimensions in Gleason's theorem not allowing of unambiguously defining the probability of any linear subspace of a qubit. Particularly, this means that the probability of a single qubit being considered as a subspace of itself cannot be unambiguously defined. If there are at least two rolls equivalent to two different qubits, Gleason's theorems states the existence of a single probability associable with any linear subspace.

This seems to be a paradox, but can be interpreted as follows:

The space consisting only of any single elementary event does not satisfy Kolmogorov's axioms in fact: It does not define any measure or probability assignable to that event equivalent to the space unambiguously. A second event (choice) is necessary in order to define unambiguously the measure of the former, single one. Indeed its probability is always identical to 1, but this cannot determine any corresponding measure $\mu_{0}$ unambiguously since $\frac{\mu_{e}}{\mu_{s}}=\frac{\mu_{0}}{\mu_{0}}=1$ for any $\mu_{0}$. Here $\mu_{e}, \mu_{s}=\mu_{0}$ are the measures of both event and space.

One might object that the single roll of the real dice is unambiguously defined, but there is in fact another event (choice) to be chosen the given dice having six sides (alternatives). In general, the dice can have any number of "sides" and any geometrical form including some irregular shape so that the alternatives ("sides") not to be equally probable.

If the number of the "sides" of the dice converges to infinity, the shape will converge to the surface of a ball, i.e. a sphere, and the dice itself to a qubit. Indeed the qubit defined as usual is isomorphic to a unit ball, within which two points are chosen: the one within the ball corresponding to the one coefficient (whether $\alpha$ or $\beta$ ), and the other on its surface corresponding to both $\alpha$ and $\beta$. Furthermore the choice among two continua is equivalent to that among a single one because any continuum is an infinite set. Thus the shape of God's dice is that of a unit ball, a qubit.

Consequently, the exception for two dimensions in Gleason's theorem is a necessary condition for any qubit to be considered as an elementary event and thus as the result rolling God's dice. 
III GOD'S DIE, GLEASON'S THEOREM AND AN IDEA FOR A SHORT PROOF OF FERMAT'S LAST THEOREM

Fermat's marginal is one of the most famous notes ever written:

Cubum autem in duos cubos, aut quadrato-quadratum in duos quadrato-quadratos, et generaliter nullam in infinitum ultra quadratum potestatem in duos eiusdem nominis fas est dividere cuius rei demonstrationem mirabilem sane detexi. Hanc marginis exiguitas non caperet $^{7}$ (Nagel 1951: 252).

Andrew Wiles (1995) managed to prove that theorem however on more than one hundred journal pages still recently and utilizing many of the last achievements of mathematics.

Fermat's marginal continues to call for a short proof using only that mathematics known until his time.

Of course, a problem is whether Gleason theorem's can be at all represented in notions and theorems of XVII century's mathematics. Nevertheless, it seems to suggest a rather shorter way for Fermat's theorem to be proved:

1. Any three Pythagorean numbers $x, y, z$ (consequently, any Pythagorean prime $z$ ) implies a different measure. As they are an infinite set, they imply a corresponding infinite set of different finite measures.

2. Any three real numbers, not being Pythagorean numbers but satisfying the equation in the Pythagorean theorem, share one single common measure for it is necessarily infinitesimal, and any continua are of an equal power.

3. Consequently, the dimensions of one and two $(n=1,2)$, which are under the exception of Gleason's theorem, allow of an infinite set of measures necessary for the equation $x^{n}+y^{n}=z^{n}$ to be able to be satisfied for some triples of natural numbers $x, y, z$.

4. Furthermore, Gleason's theorem excludes the existence of more than one single measure for any other number of dimensions ( $n>2)$ and thus it does not allow of $x^{n}+y^{n}=z^{n}$ to hold for any triple of natural numbers $x, y, z$. The admissible one single measure is necessarily the infinitesimal one.

5. The utilized concept of measure should be much closer to the Pythagorean one implicitly suggested in the shocked the Pythagoreans incommensurability of the hypotenuse and cathetus of almost all right triangles rather than to the contemporary too generalized notion of measure:

It should suggest an equal number of steps of a procedure for any two commeasurable lengths (or any quantities). If the number of steps is different for the shortest possible procedures, the corresponding measure should be accepted as different. However if the number of necessary steps is infinite, the corresponding measure should be accepted as one and the same.

6. What remains to be proved is that concept of measure in Gleason's theorem is relevant and consistent to the above Pythagorean kind of measure. However that problem turns out to be rather

\footnotetext{
7 "It is impossible for a cube to be the sum of two cubes, a fourth power to be the sum of two fourth powers, or in general for any number that is a power greater than the second to be the sum of two like powers. I have discovered a truly marvelous demonstration of this proposition that this margin is too narrow to contain."

The translation in English is according to http://mathworld.wolfram.com/FermatsLastTheorem.html (accessed at 21.05.2015).
} 
philosophical than mathematical: Whether a space of events is one and the same where it is standalone as a set and where it is a subset of another. It seems that neither Gleason touches that question nor it in turn refers to his proof in any way ${ }^{8}$. If no, Gleason's theorem seems to imply the above Pythagorean concept of measure, and if yes, it does not.

If the former is the case, the fundamental and inherent uncertainty of God's dice having infinite number of sides is intimately linked and perhaps even equivalent to Fermat's last theorem. However one needs an additional axiom that is the case.

\section{INTERPRETATION OF THE THESIS}

Gleason's theorem cannot define any sufficient condition and thus both necessary and sufficient condition for God's dice to be namely a qubit since any exception for the Hilbert space on any field satisfies the necessary condition. Consequently, a second reflection is necessary to elucidate the meaning for the Hilbert space of quantum mechanics to be just the complex one.

God's dice need a second dimension, different from the real one of probability, in order to be able to determine unambiguously not only the probability but furthermore the energy corresponding to a certain kind of relation between two probabilities by the meditation of the fundamental Planck constant. The one of those probabilities is given by the value in the first dimension, the "real" one.

That certain kind of relation between two probabilities, the given $p_{g}$ and some other, $p_{o}$ is not reflexive, neither symmetric nor antisymmetric, if the relation is that in the quantity of information (mutual entropy) define as usual (i.e. in Boltzmann - Gibbs - Shannon) $p_{o} \ln \left(p_{g}\right) \neq p_{g} \ln \left(p_{o}\right)$. The probability $p_{o}$ can be interpreted differently, e.g. by means of $p_{g}-p_{o}$ or $\left|p_{g}-p_{o}\right|$, as the change of probability, particularly as the derivative of probability if $p_{g} \rightarrow p_{o}$, i.e. $p_{g}$ converges to $p_{o}$. Thus the probability $p_{o}$ can be understood as a limit or as an ideal image of $p_{g}$ in some other ordering or in the ideal ordering distinguishable from the real one.

Having got the dimension of information, $p_{o} \ln \left(p_{g}\right)$ cannot be less than a bit, and one bit as a physical limit corresponds to ratio of the Planck constant per a unit of time and thus to an exactly specified amount of energy (a very, very small amount in relation to the macroscopic physical quantities).

All this calls an analogy between two Einstein's famous formulas (1905), $E=m c^{2}$, and $E=h f$ :

$$
E=h I
$$

Here $I$ means not directly information, but the change of information per a unit of time, or the derivative of information to time. It can be interpreted physically as the frequency of the associated de Broglie (1925) wave specifying any quantum system and corresponding to its mass, which is obvious if one juxtaposes both Einstein's formulas above. This means that the physical quantity of mass corresponds to the flux of information passing through the present (i.e. per a unit of time).

\footnotetext{
${ }^{8}$ The definition of measure used by him is: "A measure on the closed subspaces means a function $\mu$, which assigns to every closed subspace a nonnegative real number such that if $\left\{A_{i}\right\}$ is a countable collection of mutually orthogonal subspaces having closed linear span $B$, then $\mu(B)=\sum \mu\left(A_{i}\right)$ " (Gleason 1957: 885). Whether B is a space or a subspace is not discussed and does not touch the proof.
} 


\section{GOD'S DICE (A QUBIT) AS A LAW OF CONSERVATION AND TRANSFORMATION}

A qubit as one is to wait for God's dice possesses many symmetries being as symmetric as possible at all. Any symmetry of them corresponds to some law of conservation. That of the physical quantity of action and probability (entropy and information) seems to be the most important one. It may be deduced as follows:

The numeral (whether $\alpha$ or $\beta$ ) of each "die" (correspondingly $|0\rangle$ and $|1\rangle$ ) is a complex number such that the squire of its module is interpretable as the probability of measuring (observing, taking place) the physical quantity determined in its phase (e.g. energy as in Section IV). Then, the other numeral (correspondingly $\alpha$ and $\beta$ ) corresponds to the probability for the same quantity not to be measured (observed, taken place). If one multiplies as tensors that qubit by the conjugate qubit, i.e. by the corresponding qubit of the conjugate, and takes into account that $|\alpha|^{2}+|\beta|^{2}=1$ (or 0 if the corresponding $C_{n}, C_{n+1}$ are both 0 ), the equation $\hbar\left\{|\alpha|^{2}+|\beta|^{2}\right\}=S$, where $S$ is the quantity of action for a pair of conjugate qubits, can be inferred. If the case is that, one can use the metaphor that a qubit is a "squire root" of a bit since the tensor product of two conjugate qubits is equivalent to the product of a bit and the Planck constant.

The physical meaning should be that the physical quantity of action and the probability of an element or a state (respectively, entropy or information of the entire system) are conserved only transforming into each other.

In particular, this implies the conservation of action rather than of energy. In fact, the generalization of conservation from energy to action has been coined already in general relativity and statistic thermodynamics. This means that the elementary units of time can be different in one and the same point of space-time in relation to a part and to the system as a whole.

God's dice demonstrate the unity and transition between the physical (i.e. material) action of a part and its disposition among the whole by meditation of probabilities (entropy or information), which are rather nonmaterial and mathematical.

\section{CONCLUSION}

God's dice turn out to be a qubit and thus having the shape of a unit ball. Any item in the universe as well the universe itself is both infinitely many rolls and a single roll of that dice for it has infinitely many "sides". Thus both the smooth motion of classical physics and the discrete motion introduced in addition by quantum mechanics can be described uniformly correspondingly as an infinite series converges to some limit and as a quantum jump directly into that limit. The second, imaginary dimension of God's dice is interpretable as energy and as the velocity of information change between two probabilities in both series and jump. 


\section{REFERENCES:}

Aspect, Alain, Philippe Grangier, and Gérard Roger (1981) "Experimental tests of realistic local theories via Bell's theorem," Physical Review Letters 47 (7): 460-463.

Aspect, Alain, Philippe Grangier, and Gérard Roger (1982) "Experimental Realization of Einstein-Podolsky-Rosen-Bohm Gedanken Experiment: A New Violation of Bell's Inequalities," Physical Review Letters 49 (2): 91-94.

Bell, John (1964) “On the Einstein - Podolsky - Rosen paradox," Physics (New York) 1 (3): 195200.

Bianchi, Massimiliano Sassoli de (2013) "Quantum dice," Annals of Physics 336 (7): 56-75.

Born, Max (1926) "Zur Quantenmechanik der Stoßvorgänge," Zeitschrift für Physik 37 (12): 863-867.

Breshears, Gerry (2008) Does God play dice with the universe? Portland, Oregon, Theological Research Exchange Network (TREN).

Broglie, Louis de (1925) "Recherches sur la théorie des quanta," Thesis (Paris), 1924, Annales de Physique (Paris, 10-ème série) 3: 22-128.

Chamberlain, Paul H. (1987) Does God play dice? The implications of quantum mechanics, Newberg, Oregon, George Fox College.

Clauser, John F. and Michael A. Horne (1974) "Experimental consequences of objective local theories," Physical Review D 10 (2): 526-535.

Clegg, Brian (2014) Dice world: science and life in a random universe, London, Icon.

Einstein, Albert (1905) "Ist die Trägheit eines Körpers von seinem Energieinhalt abhängig?" Annalen der Physik 18 (13): 639-641.

Einstein, Albert (1905) "Über einen die Erzeugung und Verwandlung des Lichtes betreffenden heuristischen Gesichtspunkt," Annalen der Physik 17 (6): 132-148.

Einstein, Albert (1926) "Letter to Max Born, 16 December 1926," in: Albert Einstein Max Born Briefwechsel 1916 - 1955 (kommentiert von Max Born). München: Nymphenburger Verlagshandlung, 1969, pp. 129-130.

Einstein, Albert, Boris Podolsky, and Nathan Rosen (1935) "Can Quantum-Mechanical Description of Physical Reality Be Considered Complete?" Physical Review 47 (10): 777-780.

Gleason, Andrew (1957) "Measures on the Closed Subspaces of a Hilbert Space," Journal of Mathematics and Mechanics 6 (6): 885-893.

Halpern, Paul (2015) Einstein's dice and Schrödinger's cat: how two great minds battled quantum randomness to create a unified theory of physics, New York, "Basic books".

Hilbert, David (1900) "Mathematische Probleme," in: Nachrichten der Königlichen Gesellschaft der Wissenschaften zu Göttingen, mathematisch-physikalische Klasse 3: 253-297.

Hilbert, David (1902) "Mathematical problems," Bulletin of the American Mathematical Society, 8 (10): 437-479.

Kolmogorov, Andrey (1933) Grundbegriffe der Wahrscheinlichkeitsrechnung, Berlin: Julius Springer

Koperski, Jeffrey (2000) "God, chaos, and the quantum dice," Zygon 35 (3): 545-559.

Montwill, Alex and Ann Breslin (2012) The quantum adventure: does God play dice? London, Imperial College Press.

Nagel, Trygve (1951) Introduction to number theory, Stockholm, Almqvist \& Wirksell. 
Peña, Luis de la and Ana María Cetto (1996) The quantum dice: an introduction to stochastic electrodynamics, Fundamental theories of physics, vol. 75, Dordrecht/ Boston, Kluwer Academic.

Ponomarev, Leonid Ivanovich (1993) The quantum dice, Bristol, Philadelphia, Institute of Physics Publishing.

Saunders, Nicholas T. (2000) "Does God cheat at dice? Divine action and quantum possibilities," Zygon, 35 (3): 517-544.

Schrödinger, Erwin (1935) "Die gegenwärtige situation in der Quantenmechanik," Die Naturwissenschaften 23 (48): 807-812; 23 (49): 823-828; 23 (50): 844-849.

Shiang, David A. (2008) God does not play dice: the fulfillment of Einstein's quest for law and order in nature, Delhi, Pustak Mahal.

Stewart, Ian (2002) Does God play dice? The new mathematics of chaos, Malden, Mass.: Blackwell.

Wiles, Andrew (1995) "Modular Elliptic Curves and Fermat's Last Theorem," Annals of Mathematics 141 (3): 443-551. 